UTHEP-376

UT-CCP-32

MPI-PhT/97-77

December 1997

\title{
The domain-wall model in an asymptotic-free dynamics
}

\author{
S. Aoki ${ }^{a, b *}$ and K. Nagai ${ }^{c \dagger}$ \\ a Institute of Physics, University of Tsukuba, Tsukuba, Ibaraki 305, Japan \\ ${ }^{b}$ Max-Planck-Institute für Physik, Föhringer Ring 6, D-80805 München, Germany \\ ${ }^{c}$ Center for Computational Physics, University of Tsukuba, Tsukuba, Ibaraki 305, Japan
}

(August 11, 2018)

\begin{abstract}
We investigate a possibility that the rough gauge problem, which have appeared to be a main reason for failures of lattice chiral gauge theories, is cured by an asymptotic-free dynamics. Taking the domain-wall model in $2(+1)$ dimensions with $\mathrm{SU}(2)$ gauge group, we carry out the quenched simulation of gauge fields in the extra dimension. By studying fermion spectra in several volumes, we show that the chiral zero modes exist on the wall without having the spontaneous symmetry breaking thanks to the asymptotic-free dynamics. This result suggests that the rough gauge problem is solved in some class of lattice chiral fermions as well as in 4 dimensions if an asymptotic-free dynamics is incorporated.
\end{abstract}

11.15Ha, 11.30Rd, 11.90.+t

Typeset using REVTEX

*aoki@mppmu.mpg.de

${ }^{\dagger}$ nagai@rccp.tsukuba.ac.jp 


\section{INTRODUCTION}

A definition of lattice chiral gauge theories is one of the long-standing problems in the lattice field theory, in spite of its success as the non-perturbative calculational method for vector gauge theories such as QCD. The difficulty is related to the no-go theorem [1], which proves, under the mild conditions, that it is impossible to define chirally invariant lattice fermion without species doubling. Despite many attempts to put chiral gauge theories on the lattice for many years, so far none of them has been proven to work successfully.

Through analyses of many unsuccessful attempts [2 6], however, a common reason of failures has been recently recognized, called the "rough gauge problem" [6], which we will explain below. A proposed lattice fermion action for one fermion field (in an anomalous representation) necessarily breaks gauge invariance, to reproduce the chiral gauge anomaly in the weak gauge coupling limit, though it may be cancelled by the contribution from other fermion fields. Because of this explicit gauge breaking, the gauge degree of freedom, which varies independently at each site, cannot be gauged away and thus interacts with the fermion, so that the fermion is not a free field even in the weak gauge coupling limit. It is now widely accepted that the roughness of the gauge degree of freedom is an essential reason for failure of the proposals such as the Wilson-Yukawa model [3], the U(1) original domain-wall model [4] and its variant [5], though the detail of the failure depends on the proposal.

By adding the kinetic term for the gauge degree of freedom to the action, one can make it smooth enough to have a desired chiral fermion spectrum. It has been shown in 4 dimensional models that the dynamics of this smoothed gauge degree( = scalar field) simultaneously leads to the condensation of the scalar field, which makes the gauge field massive in lattice unit, so that no chiral gauge theory is defined in the continuum limit. This unsatisfactory correspondence that symmetric phase - vector spectrum or Higgs phase - chiral spectrum seems to be established almost for all models.

Recently, it is, however, claimed that this correspondence is not true and thus the rough gauge problem might be solved if the dynamics of the gauge degree of freedom becomes asymptotic-free [7]. It is actually shown in the spin wave calculation that the rough configuration decouples from the fermion in the overlap formula [8].

In this paper we pursue this possibility further, using the quenched numerical simulation of the original domain-wall model, where the gauge degree of freedom corresponds to the gauge field in the extra dimension. Previously we studied the original domain-wall model with $\mathrm{U}(1)$ gauge group both in $2(+1)$ and $4(+1)$ dimensions, whose gauge field in the extra dimension has the asymptotic non-free dynamics and indeed found no chiral zero mode in the symmetric phase. In contrast, we consider here the $\mathrm{SU}(2)$ gauge group in $2(+1)$ dimensions, where the gauge field in the extra dimension is equivalent to an asymptotic-free $\mathrm{SU}(2) \times$ $\mathrm{SU}(2)$ non-linear $\sigma$ model. Therefore there is only one phase, symmetric phase, in the model. By the quenched Monte-Carlo simulation of the model we investigate an existence of chiral zero modes near and below the scaling region of the model. This paper is organized as follows. We explain the original domain-wall model in Sec.[1. In Sec.III the method of our analyses is explained, then the results are shown. Our conclusion and discussion are given in Sec.[V. 


\section{THE DOMAIN-WALL MODEL}

The original domain-wall model is the $2 k(+1)$-dimensional Wilson fermion whose mass term has a kink-like shape in the extra dimension, vectorically interacting with the $2 k(+1)$ dimensional gauge fields. The action of the original model, reformulated in Ref. [9] in terms of a $2 k$-dimensional theory, are given as

$$
S=S_{G}+S_{F},
$$

where $S_{G}$ is the gauge action and $S_{F}$ is the fermionic action. $S_{G}$ is given by

$$
S_{G}=\beta \sum_{n, \mu>\nu} \sum_{s} \operatorname{Re} \operatorname{Tr}\left[U_{\mu \nu}^{P}(n, s)\right]+\beta_{s} \sum_{n, \mu} \sum_{s} \operatorname{Re} \operatorname{Tr}\left[U_{\mu D}^{P}(n, s)\right],
$$

where $\mu, \nu$ run from 1 to $2 k, n$ is a $2 k$-dimensional lattice point, and $s$ is a coordinate of an extra dimension. $U_{\mu \nu}^{P}(n, s)$ is a $2 k$-dimensional plaquette and $U_{\mu D}^{P}(n, s)$ is a plaquette containing two link variables in the extra direction $(D=2 k+1)$. $\beta$ is the inverse gauge coupling for the plaquette $U_{\mu \nu}^{P}$ and $\beta_{s}$ is the one for the plaquette $U_{\mu D}^{P}$. In general, we can take $\beta \neq \beta_{s}$. The fermionic action $S_{F}$ on the Euclidean lattice is given by

$$
\begin{aligned}
S_{F}= & \frac{1}{2} \sum_{n \mu} \sum_{s} \bar{\psi}(n, s) \gamma_{\mu}\left[U_{\mu}(n, s) \psi(n+\mu, s)-U_{\mu}^{\dagger}(n-\mu, s) \psi(n-\mu, s)\right] \\
& +\sum_{n} \sum_{s, t} \bar{\psi}(n, s)\left[M_{0} P_{R}+M_{0}^{\dagger} P_{L}\right]_{s, t} \psi(n, t) \\
& +\frac{1}{2} \sum_{n \mu} \sum_{s} \bar{\psi}(n, s)\left[U_{\mu}(n, s) \psi(n+\mu, s)+U_{\mu}^{\dagger}(n-\mu, s) \psi(n-\mu, s)-2 \psi(n, s)\right],
\end{aligned}
$$

where $s, t$ are an extra coordinates, $P_{R / L}=\frac{1}{2}\left(1 \pm \gamma_{2 k+1}\right)$,

$$
\left\{\begin{array}{l}
\left(M_{0}\right)_{s, t}=U_{D}(n, s) \delta_{s+1, t}-a(s) \delta_{s, t} \\
\left(M_{0}^{\dagger}\right)_{s, t}=U_{D}^{\dagger}(n, s-1) \delta_{s-1, t}-a(s) \delta_{s, t} .
\end{array}\right.
$$

Here $U_{\mu}(n, s), U_{D}(n, s)$ are link variables connecting a site $(n, s)$ to $(n+\mu, s)$ or $(n, s+1)$, respectively. Because of a periodic boundary condition in the extra dimension, $s, t$ run from $-L_{s}$ to $L_{s}-1$, and $a(s)$ is given by

$$
\begin{aligned}
a(s) & =1-m_{0} \operatorname{sign}\left[\left(s+\frac{1}{2}\right) \operatorname{sign}\left(L_{s}-s-\frac{1}{2}\right)\right] \\
& = \begin{cases}1-m_{0}\left(-\frac{1}{2}<s<L_{s}-\frac{1}{2}\right) \\
1+m_{0}\left(-L_{s}-\frac{1}{2}<s<-\frac{1}{2}\right),\end{cases}
\end{aligned}
$$

where $m_{0}$ is the height of the domain wall mass. It is easy to check that the above fermionic action is identical to the one in $2 k(+1)$ dimensions, proposed by Kaplan [2,9]. This model describes chiral fermions for smooth back-ground gauge fields in perturbation theory [10. We studied the weak coupling limit, where the physical gauge coupling constant $g \rightarrow 0$. In this case, all gauge fields in the physical dimensions can be gauged away, while the gauge field in the extra dimension is still dynamical and its dynamics is controlled by $\beta_{s}$. Instead of the gauge degrees of freedom in the edge of the waveguide $(2 k+1)$ th component of gauge 
link variables represent roughness of $2 k$-dimensional gauge fields. An important question is whether the chiral zero mode on the domain wall survives in the presence of this rough dynamics.

In this weak coupling limit, the dynamics of the gauge field is equivalent to $2 k$ dimensional scalar model with $2 L_{s}$ independent copies where $2 L_{s}$ is the number of sites in the extra dimension, as following way:

$$
S_{G}=\beta_{s} \sum_{s} \sum_{n, \mu}\left\{1-\operatorname{Re} \operatorname{Tr}\left[V(n, s) V^{\dagger}(n+\mu, s)\right]\right\}
$$

where $V(n, s)=U_{D}(n, s)$. If the gauge group is $\mathrm{SU}(\mathrm{N})$, the above action is equivalent to an $\mathrm{SU}(\mathrm{N}) \times \mathrm{SU}(\mathrm{N})$ non-linear $\sigma$ model at each $s$, therefore it is asymptotic-free in 2 dimensions and has only a symmetric phase for all $\beta_{s}$. Larger $\beta_{s}$ smoother $V(n, s)$ without having non-zero condensation of $V(n, s)$. We can increase $\beta_{s}$ as much as we want till the chiral zero modes will appear on the wall. If it appears at finite $\beta_{s}$ we can conclude that the chiral fermion exists in the symmetric phase, so the model passes the first test toward the construction of lattice chiral gauge theories. In this paper we take SU(2) as the gauge group and investigate it in $g \rightarrow 0$ limit by the quenched simulation.

\section{ANALYSES AND RESULTS}

The method of simulation and analysis is essentially the same in Ref. [4] except the gauge group. In order to determine the choices of $\beta_{s}$ for the fermion propagator calculation, we first calculate the correlation length of the scalar field $V(n, s)$ and the result is given in Fig. 1 as a function of $\beta_{s}$. Form this figure we estimate that the scaling region, where the correlation length grows exponentially in $\beta_{s}$, begins around $\beta_{s}=2.0$. This estimation is consistent with the one estimated from the susceptibility [11. We also see from the data between $L=16$ and 32 that the finite size effect in the correlation length is negligible at $\beta_{s} \leq 2.0$ for $L=16 \equiv L_{\min }$. The relation expected from consistency that $\xi<8=L_{\min } / 2$ also holds at $\beta_{s} \leq 2$.

From the above result on the correlation length we decide to calculated the fermion propagators at $\beta_{s}=1.0$ and 2.0 for several values of $m_{0}$ on different sizes of lattices, using a quenched approximation. The fermion mass in the 2-dimensional theory is extracted from the fermion propagator within the $s=0$ layer as follow 2 . The fermion propagator $G(p)_{s t}$ in the 2 dimensional momentum space and the coordinate of the extra dimension is written as

$$
G(p)_{s, t}=\left[\left(-i \sum_{\mu} \gamma_{\mu} \bar{p}_{\mu}+M\right) G_{R}(p)\right]_{s, t} P_{L}+\left[\left(-i \sum_{\mu} \gamma_{\mu} \bar{p}_{\mu}+M^{\dagger}\right) G_{L}(p)\right]_{s, t} P_{R} .
$$

\footnotetext{
${ }^{1}$ Note that the notation of the coupling constant in their study is twice smaller than ours.

${ }^{2}$ The details of this method and the notation are explained in Ref. 狛. However, note that $G_{L}\left(G_{R}\right)$ here is denoted $G_{R}\left(G_{L}\right)$ in Ref. [4]
} 
Extracting $G_{L}(p)_{s=t=0}$ and $G_{R}(p)_{s=t=0}$ and extrapolate them to $p=0$ we obtain the (effective) fermion mass $m_{f}$ as

$$
\begin{aligned}
\left(m_{f}^{L}\right)^{2} & =\lim _{p \rightarrow 0} \frac{1}{G_{L}(p)_{s=t=0}} \\
\left(m_{f}^{R}\right)^{2} & =\lim _{p \rightarrow 0} \frac{1}{G_{R}(p)_{s=t=0}}
\end{aligned}
$$

where $L(R)$ for $m_{f}$ stands for the left-handed(right-handed). We take a periodic boundary condition in the first- and the extra-directions and anti-periodic boundary in the seconddirection to avoid possible singularity of a massless fermion. The fermion propagator is obtained from the average over 50 configurations and the errors are estimated by jack-knife method with an unit bin size.

In Fig. 2 the fermion mass extracted in this way is plotted as a function of $m_{0}$ at $\beta_{s}=1.0$ and 2.0 on $L \times 32 \times 2 L_{s}$ lattices with $L=8,16,32$ and $L_{s}=8$. This figure shows that the right-handed mode becomes massless when $m_{0}$ is grater than some critical value: $m_{0}^{c}$. For example, $m_{0}^{c} \sim 0.8-0.95$ at $\beta_{s}=1.0$ and $m_{0}^{c} \sim 0.4-0.6$ at $\beta_{s}=2.0$. On the other hand, the left-handed mode are massive at all $m_{0}$. It also shows that the finite size effect is small between $L=16$ and 32, though some effect is seen, in particular at $\beta=1.0$, for $L=8$. This lattice size dependence of fermion masses is consistent with the one of the correlation length of the scalar field, and is very different from the size dependence of the fermion mass for asymptotic non-free cases [4]. These results strongly indicate that the chiral fermion

spectrum observed on $L=16$ and 32 remains in the infinite volume limit: the chiral zero modes exist on the wall in the infinite lattice volume of 2-dimensional physical space-time.

In addition to the analysis on the fermion masses, we carry out the mean-field analysis, which has been shown to be successful to explain not only the existence of the critical value of the domain wall mass, but also the behavior of the fermion propagator [i]. We fit the measured propagator with the form of the mean-field propagator obtained by replacing the link variable to some unknown but constant value $z$. Note that non-zero value of $z$ means an existence of the chiral zero mode for the range that $1-z \leq m_{0}<1$. The extracted values of $z$ 's are plotted as a function of $m_{0}$ in Figs. 3 and 4 . Within relatively large errors, $z$ 's are always non-zero at both $\beta_{s}$. At $\beta_{s}=2 z$ depends very weakly on $m_{0}$ while it increases as $m_{0}$ approaches to 1 at $\beta_{s}=1$. A similar $m_{0}$ dependence to the latter case has been also seen previously for asymptotic non-free cases [4]. Since $z$ should be constant on $m_{0}$ if the mean-field approximation is exact, this $m_{0}$ dependence shows the magnitude of the error for the mean-field approximation. Again the finite size effect is small between $L=16$ and 32 , contrary to the case of the asymptotic non-free dynamics [四], and no indication that $z$ goes to zero as volume increases is observed. In conclusion the mean-filed analysis also supports, at least at $\beta_{s}=2$, the existence of the chiral zero mode on the wall in the infinite volume limit.

\section{CONCLUSIONS AND DISCUSSIONS}

In this paper we numerically investigate an existence of chiral zero modes of the original domain-wall model in the presence of asymptotic-free dynamics in $2(+1)$ dimensions using 
the quenched simulation. The result of the fermion mass on $16 \times 32 \times 16$ and $32 \times 32 \times 16$ suggests that the chiral zero mode observed on the domain-wall at $s=0$ will survive in the infinite volume limit. The mean-field analysis of the fermion propagator also supports this conclusion.

Although the positive indication is obtained for the construction of lattice chiral gauge theories via the domain-wall model with asymptotic-free dynamics, we have to understand a difference between an asymptotic-free and an asymptotic non-free cases more deeply. It is usually thought that the vacuum expectation value of the scalar field controls the existence of the chiral zero mode in the model: If non-zero the zero mode exists, if not it does not. In terms of the mean field analysis, $z$ is supposed to correspond to the vacuum expectation value. This is a crucial property for the failure of the domain-wall model in the symmetric phase, and this seems true for an asymptotic non-free dynamics [4]. However the result of this paper shows that this does not hold anymore for the asymptotic-free dynamics. Then, what controls the existence of zero modes? What corresponds to $z$ ? It is likely that some function of the correlation length does it. Since the phase is unique for the $\mathrm{SU}(2) \times \mathrm{SU}(2)$ non-linear $\sigma$ model in 2 dimensions, the function should be analytic in the correlation length, so that it can vanish only on some points of $\beta_{s}$, but not in the non-zero region of $\beta_{s}$. It is more likely that it vanishes only at $\beta_{s}=0$. If this is true the zero mode exists for all $\beta_{s}$ except $\beta_{s}=0$. However since the scalar field near $\beta_{s}=0$ is very rough, the dynamics is very similar to that in the symmetric phase of the asymptotic non-free model. Therefore an existence of zero mode near $\beta_{s}=0$ may imply an existence of the zero mode also in the symmetric phase of the asymptotic non-free model. This seems inconsistent with the previous numerical results [4]. The one of solution to this puzzle is perhaps that the allowed range of the domain-wall mass $m_{0}$ for the zero mode $\left(: m_{c} \leq m_{0} \leq 1\right)$ is too narrow to be detected in the numerical simulation at the accuracy of Ref. [4]. This point should be clarified, in order to establish the existence of the chiral zero mode of the asymptotic-free model without doubt.

Since scalar field theories with second derivative terms are asymptotic non-free in 4 dimensions, higher derivative terms have to be introduced to make them asymptotic-free [7]. Since, in terms of gauge fields, these higher derivative terms correspond to the gauge fixing terms, the solution to the rough gauge problem by the asymptotic-free dynamics in 4 dimensions is very similar to the recent proposal to the problem by the gauge fixing for the $\mathrm{U}(1)$ theory [12]. The relation between the two should be understood.

If the rough gauge problem of the chiral zero mode in the domain-wall model can indeed be solved by an asymptotic-free dynamics, the rough gauge problems appeared in other models such as the Wilson-Yukawa model and the Waveguide model are also resolved by the same dynamics. Research in this direction is also necessary in order to define lattice chiral gauge theories ultimately.

\section{ACKNOWLEDGEMENTS}

Numerical calculations for the present work have been carried out at Center for Computational Physics and on VPP500/30 at Science Information Center, both at University of Tsukuba. This work is supported in part by the Grants-in-Aid of the Ministry of Education (Nos. 08640349, 09246206). 


\section{REFERENCES}

[1] H. B. Nielsen and M. Ninomiya, Nucl. Phys. B185, 20 (1981); erratum: ibid. B195, 541 (1982); Nucl. Phys. B193, 173 (1981); Phys. Lett. B105, 219 (1981);

L. H. Karsten, Phys. Lett. B104, 315 (1981).

[2] D. B. Kaplan, Phys. Lett. B288, 342 (1992).

For a recent review of domain-wall model, see, K. Jansen, Phys. Rep. 273 ,1 (1996) .

[3] W. Bock, A. K. De and J. Smit, Nucl. Phys. B388, 243 (1992);

M. F. L. Golterman, D. N. Petcher and E. Rivas, Nucl. Phys. B377, 405 (1992);

W. Bock, A. K. De, E. Focht and J. Smit, Nucl. Phys. B401, 481 (1993);

S. Aoki, H. Hirose and Y. Kikikawa Int. J. Mod. Phys. A9, 4129 (1994) .

[4] S. Aoki and K. Nagai, Phys. Rev. D53, 5058 (1996); Phys. Rev. D56, 1121 (1997).

[5] M. F. L. Golterman, K. Jansen, D. N. Petcher, and J. C. Vink, Phys. Rev. D49, 1606 (1994);

M. F. L. Golterman and Y. Shamir, Phys. Rev. D51, 3026 (1995).

[6] D. N. Petcher, Nucl. Phys. B (Proc. Suppl.) 30, 50 (1993);

Y. Shamir, Nucl. Phys. B (Proc. Suppl.)47, 212 (1996) .

[7] Y. Kikukawa, KUNS-1445, hep-lat/9705024 (In two dimensions); KUNS-1446, heplat/9707010 (In four dimensions).

[8] R. Narayanan and H. Neuberger, Nucl. Phys. B412, 574 (1994).

[9] R. Narayanan and H. Neuberger, Phys. Lett. B302, 62 (1993).

[10] S. Aoki and H. Hirose Phys. Rev. D49, 2604 (1994);

S. Aoki and R. B. Levien, Phys. Rev. D51, 3790 (1995).

[11] Y. Kikukawa and S. Miyazaki, Prog. Theor. Phys. 96, 1189 (1996).

[12] Y. Shamir, TAUP-2306-95, hep-lat/9512019; Nucl. Phys. B (Proc. Suppl.) 53, 664 (1997);

M. Golterman and Y. Shamir, Phys. Lett. B399, 148 (1997);

W. Bock, M. Golterman and Y. Shamir, TAUP-2447-97, hep-lat/9708019; HU-EP-97-

64, hep-lat/9709113; HU-EP-97-65, hep-lat/9709115; TAUP-2454-97, hep-lat/9709154. 


\section{FIGURES}

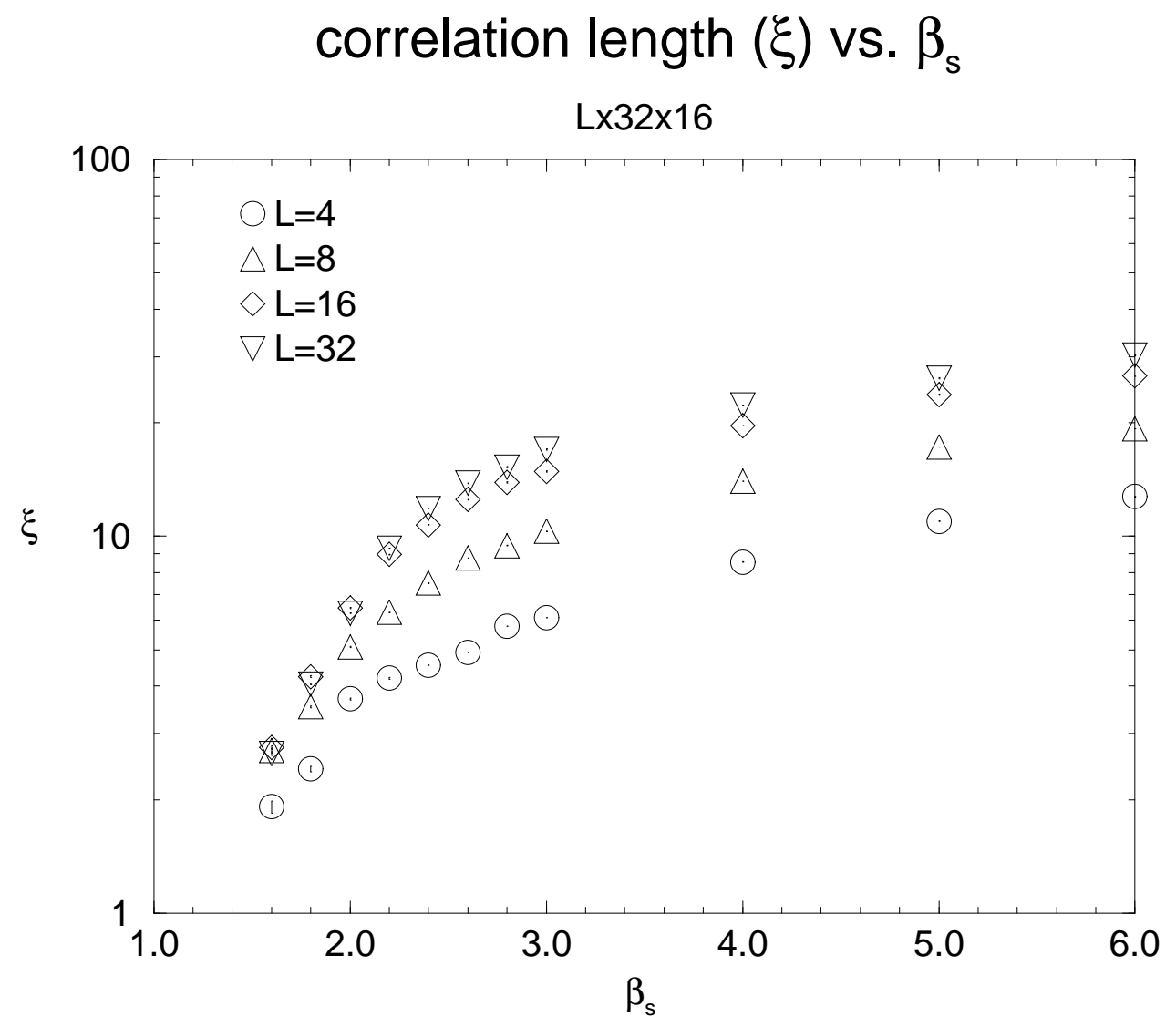

FIG. 1. Correlation length $(\xi)$ vs. $\beta_{s}$ on a $L \times 32 \times 16$ lattices with $L=4$ (circles) , 8 (up triangles) , 16(diamonds) and 32(down triangles). The error bar is smaller than the symbol. 

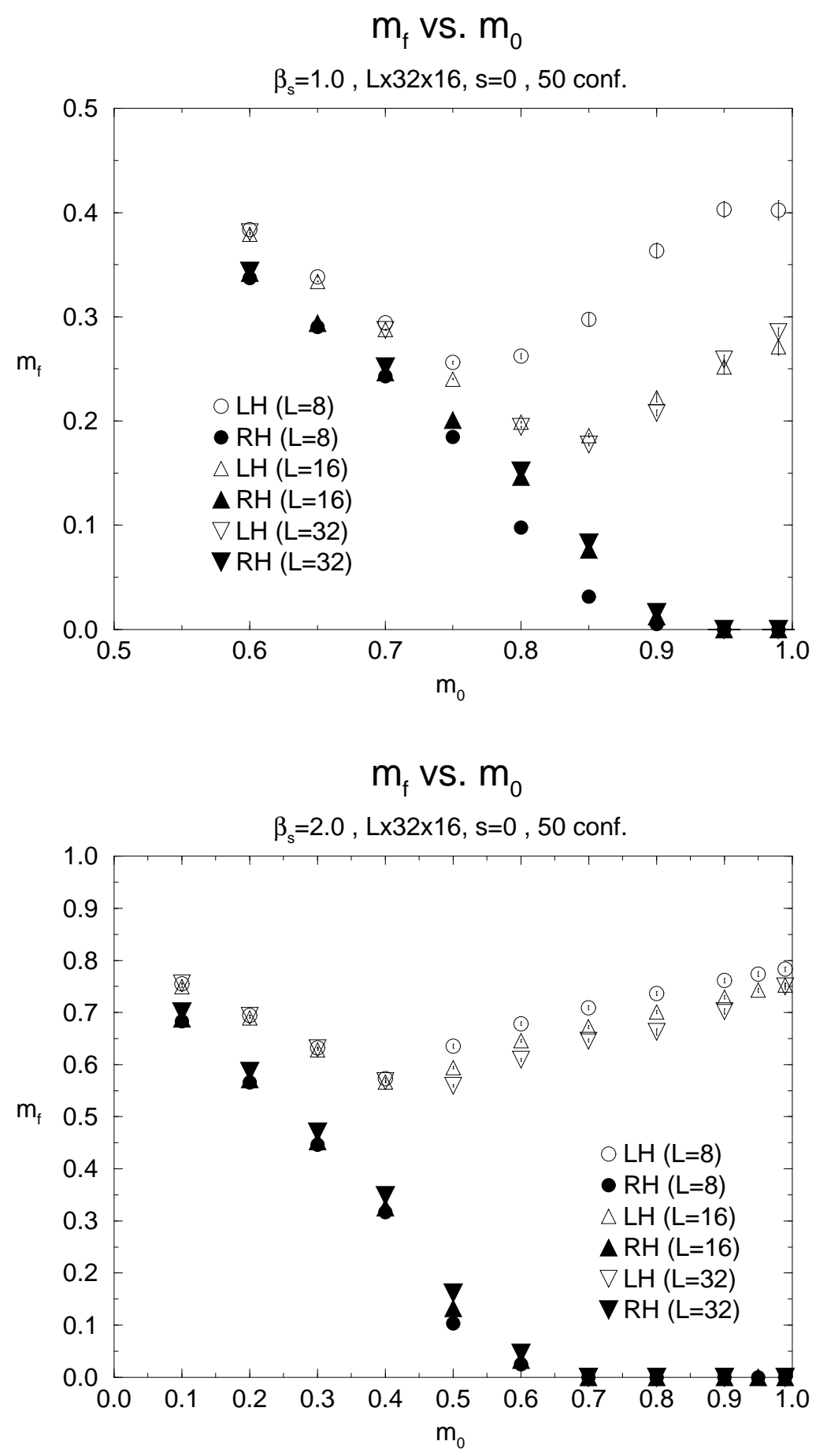

FIG. 2. $m_{f}$ vs. $m_{0}$ at $\beta_{s}=1.0$ and 2.0 on a $L \times 32 \times 16$ lattices with $L=8$ (circles), 16 (up triangles) and 32 (down triangles) obtained form the fermion propagator on the domain wall at $s=0$, for the right-handed fermion (solid symbols) and the left-handed fermion (open symbols). 

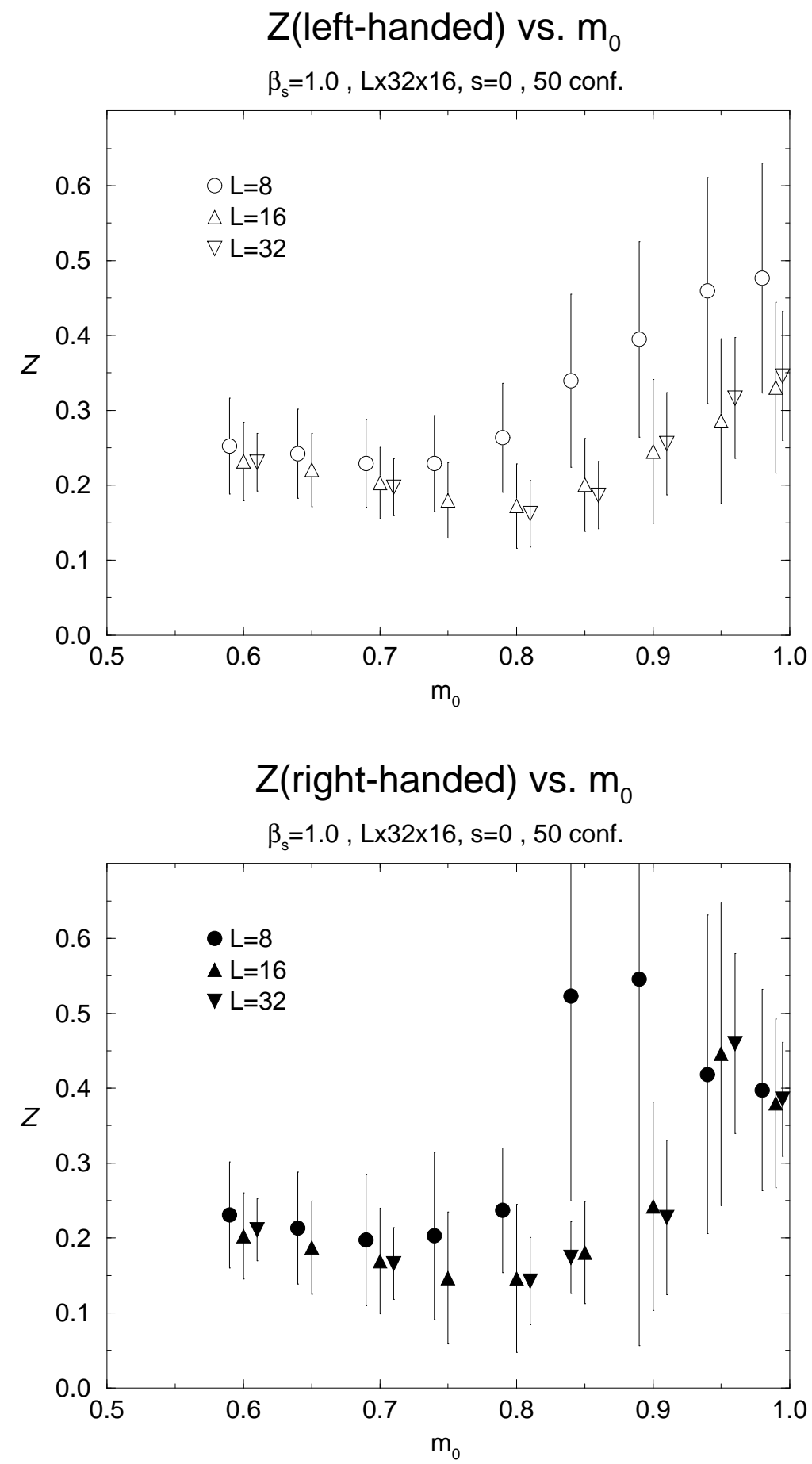

FIG. 3. (a) $z$ (left-handed) vs. $m_{0}$ at $\beta_{s}=1.0$ (b) $z$ (right-handed) vs. $m_{0}$ at $\beta_{s}=1.0$ on $L \times 32 \times 16$ lattice with $L=8$ (circles), 16(up triangles) and 32(down triangles), obtained form the fermion propagator on the domain wall at $s=0$. 

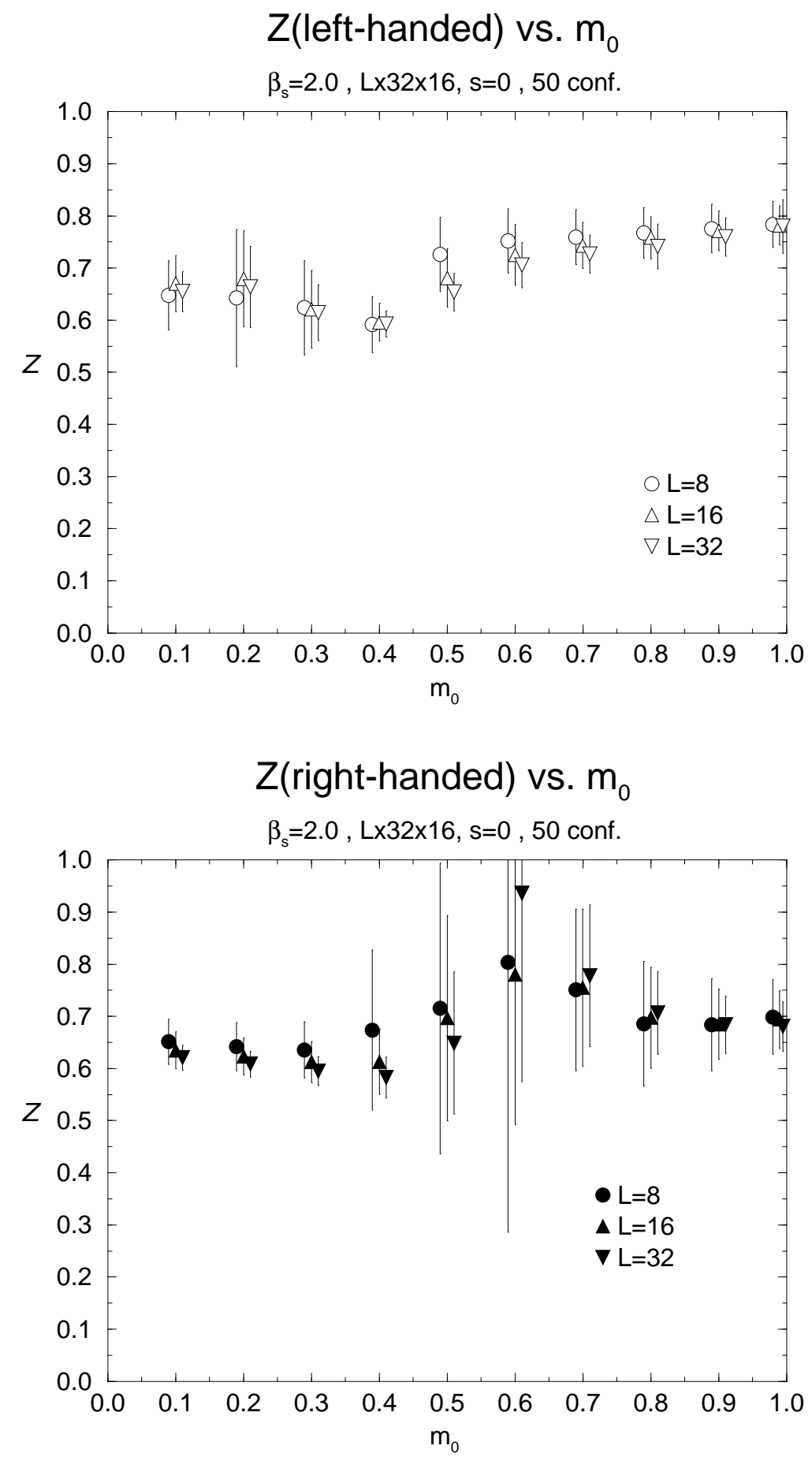

FIG. 4. (a) $z$ (left-handed) vs. $m_{0}$ at $\beta_{s}=2.0$ (b) $z$ (right-handed) vs. $m_{0}$ at $\beta_{s}=2.0$ on $L \times 32 \times 16$ lattice with $L=8$ (circles), 16(up triangles) and 32(down triangles) obtained form the fermion propagator on the domain wall at $s=0$. 